\title{
Ultrasound cardiogram-based diagnosis of cardiac hypertrophy from hypertension and analysis of its relationship with expression of autophagy-related protein
}

\author{
Sha $\mathrm{Li}^{1}$, Lina Zhao ${ }^{1}$, Bei Zhang ${ }^{1}$, Yao Yuan ${ }^{1}$, Hongjuan $\mathrm{Cao}^{1}$, Zhenqiu $\mathrm{Yu}^{2}$ \\ ${ }^{1}$ Ultrasonic Center, Guizhou Medical University, Guiyang, China; ${ }^{2}$ Hypertension Department, Guizhou Medical University, Guiyang, China \\ Contributions: (I) Conception and design: S Li, Z Yu; (II) Administrative support: L Zhao; (III) Provision of study materials or patients: S Li, L Zhao, \\ B Zhang, Y Yuan, H Cao; (IV) Collection and assembly of data: All authors; (V) Data analysis and interpretation: S Li, B Zhang, Y Yuan, H Cao, Z \\ Yu; (VI) Manuscript writing: All authors; (VII) Final approval of manuscript: All authors. \\ Correspondence to: Zhenqiu Yu. Guizhou Medical University, No. 9 Beijing Road, Guiyang, China. Email: yuzhenqiuazz@163.com.
}

Background: Ultrasound cardiogram is commonly used in the diagnosis of cardiac hypertrophy from hypertension. This study aimed to investigate the correlation between the occurrence of cardiac hypertrophy from hypertension with the expression of autophagy-related protein 9A (ATG9a).

Methods: In this study, 168 patients with hypertension in the Guizhou Medical University from February 2020 to September 2021 were selected. The patients were divided into an experimental group (cardiac hypertrophy group) and a normal group according to the results of ultrasound cardiogram examination, and serum ATG9a levels in the two groups were detected. The association between ATG9a and cardiac hypertrophy from hypertension and the relationship between serum ATG9a and ultrasound cardiogram indicators were analyzed. And a receiver operating characteristic (ROC) curve was drawn to analyze the value of ATG9a in the diagnosis of cardiac hypertrophy from hypertension.

Results: The results showed that there were no significant differences in age, diastolic blood pressure, hypertension course, body mass index (BMI), smoking history, and drinking history between the experimental and normal groups $(\mathrm{P}>0.05)$. Binary logistic regression analysis showed that compared with the normal control group, ATG9a increased significantly $(\mathrm{P}<0.05)$ and systolic blood pressure decreased significantly in the experimental group. The results showed that the area under the curve (AUC) of serum ATG9a was 0.736, the sensitivity was $76.54 \%$, and the specificity was $78.42 \%$ in the diagnosis of cardiac hypertrophy from hypertension. Pearson correlation analysis showed that ATG9a was positively correlated with left ventricular posterior wall thickness (LVPWT) and interventricular septal thickness (IVST) in patients with cardiac hypertrophy from hypertension, and was negatively correlated with left ventricular ejection fraction (LVEF) $(\mathrm{P}<0.05)$.

Conclusions: Serum ATG9a may be involved in the formation of cardiac hypertrophy in hypertensive patients. Our results, which showed that serum ATG9a level increased in cardiac hypertrophy patients, were consistent with the clinical ultrasonic cardiogram diagnosis result, and ATG9a is expected to be a marker for early ultrasonic cardiogram diagnosis.

Keywords: Ultrasound cardiogram; cardiac hypertrophy; autophagy-related protein; specificity; receiver operating characteristic curve (ROC curve)

Submitted Dec 11, 2021. Accepted for publication Feb 21, 2022.

doi: 10.21037/apm-21-3936

View this article at: https://dx.doi.org/10.21037/apm-21-3936 


\section{Introduction}

At present, hypertension, as a chronic non-communicable disease, has attracted widespread attention and become a serious problem affecting global public health (1). With the improvement of people's living standards and the aging of the population, the incidence of hypertension is increasing. In China, the annual growth of hypertension can reach up to 10 million people per year. Previous studies have estimated that there will be more than 1.5 billion people suffering from hypertension globally by 2025 (2-4). Hypertension is often accompanied by other serious cardiovascular diseases, including cerebral apoplexy, myocardial infarction, heart failure, and coronary heart disease $(5,6)$. In recent years, younger patients have experienced a variety of cardiovascular and cerebrovascular diseases caused by hypertension, and the incidence has been increasing annually (7). Hypertension has become a risk factor for mortality, which has a serious impact on quality of life and also threatens the lives of patients, resulting in a considerable economic burden to both patients' families and society $(8,9)$. The treatment principles for patients with hypertension involve strict and effective control of patients' blood pressure levels, while avoiding or slowing down the occurrence of hypertension-related complications, in order to ensure the quality of life of patients $(10,11)$. Evidencebased and effective management plays a positive role in controlling the blood pressure level of hypertensive patients as well as avoiding the occurrence and deterioration of complications (12).

A long-term high blood pressure level will lead to ventricular remodeling, which is clinically manifested as ventricular wall hypertrophy, ventricular cavity enlargement, and changes in ventricular cavity geometry (13). The heart has various adaptive compensatory responses to the long-term presence of high blood pressure. Cardiac hypertrophy, as one of the compensatory responses, can reduce the tension of the ventricular wall. However, in the long term, cardiac hypertrophy will result in heart failure and lead to serious consequences (14-16). Studies have shown that among patients with hypertension, those with left ventricular hypertrophy account for about onethird, and three-quarters heart failure cases are caused by hypertension (17-19). Patients with hypertension are likely to develop cardiac dysfunction, resulting in hypertensive myocardial damage, which is most commonly manifested as cardiac hypertrophy from hypertension $(20,21)$. Cardiac hypertrophy from hypertension is the result of increasing left ventricular load caused by high blood pressure over a long period (22-24). Cardiac hypertrophy patients often have a series of pathological changes, which are mainly manifested as cardiomyocyte hypertrophy, interstitial fibrosis, and non-cardiac hyperplasia (25-27).

Ultrasound cardiogram is a diagnostic method for hypertensive cardiac hypertrophy, and is widely used in clinical practice. Its detection rate of hypertension cardiac hypertrophy is approximately $73.15 \%$ (28-30). Ultrasound cardiogram is a non-invasive detection technology that can examine the anatomical structure and functional status of the heart and its large blood vessels through the unique physical characteristics of ultrasonic short-waves (31). Since the application of ultrasound in the diagnosis of heart disease in 1954, researchers have extensively applied and improved ultrasound cardiogram. The clinical manifestations of ultrasound cardiogram are divided into three types, namely M-type ultrasound cardiogram, two-dimensional ultrasound cardiogram, and Doppler ultrasound cardiogram $(32,33)$. Although ultrasound cardiogram can temporarily meet the diagnostic needs of patients with hypertrophy caused by hypertension, its accuracy and detection rate are limited. Hence, researchers are exploring more accurate diagnostic and treatment methods (34). The relationship between cardiac hypertrophy and autophagy has not been completely clarified. It has been reported that inhibition of autophagy counteracts cardiac hypertrophy, improves myocardial remodeling, increases patient survival, and ameliorates cardiac hypertrophy (35). More than 40 autophagyrelated genes (ATG) have been identified, and according to different functions, the autophagy-related protein ATG has been classified into 4 complexes: ATG1 protein kinase complex, ATG9 complex, phosphatidylinositol 3-kinase PI3K complex, and ATG5/12/16 complex (36). It has been reported that autophagy-related protein 9A (ATG9a), as an autophagy regulatory protein, plays an important role in regulating cardiac autophagy and inhibiting cardiac hypertrophy. It is abnormally expressed in patients with cardiac hypertrophy from hypertension (37). As a multitransmembrane protein, ATG9a plays a crucial role in the formation of autolysosomes; transport of ATG9a by endosomes is a key step of the formation of autolysosomes. Investigating the expression level of ATG9a in patients with cardiac hypertrophy from hypertension may provide evidence for the diagnosis of the disease. Pathological myocardial hypertrophy is due to impairment of some of the signaling pathways associated with hypertrophy, some of which are also involved in the regulation of cellular 
autophagy. Calcium/calcium-regulated signaling pathway promotes the development of myocardial hypertrophy by activating gene transcription. The PI3K/AKT pathway acts on two downstream targets, glycogen synthase kinase- 3 and mTOR, to regulate myocardial hypertrophy, both of which are major regulators of cardiomyocyte autophagy (38). Hypertrophy is also regulated through the mitogenactivated protein kinases (MAPKs) signaling pathway, and MAPKs are also associated with cardiomyocyte autophagy and mitochondrial autophagy. Among the many growth factors that can induce cardiomyocyte hypertrophy, Ang II was confirmed to be the most important stimulator, and its receptor can regulate cardiomyocyte autophagic activity. It has been shown that interference with ATG9a expression attenuates Ang II-mediated increases in cardiomyocyte autophagic activity and hypertrophy (39).

The study aimed to investigate the application value of ultrasound cardiogram in the clinical diagnosis of cardiac hypertrophy from hypertension and the correlation between its occurrence and the expression of ATG9a. One hundred and sixty-eight hypertension patients from Guizhou Medical University were selected and divided into an experimental group (cardiac hypertrophy group) and a normal group according to the results of the ultrasound cardiogram examination. The serum ATG9a levels of patients in the two groups were detected. In this study, we innovatively linked serum ATG9a with hypertensive cardiac hypertrophy and analyzed the relationship between ATG9a and hypertension-induced cardiac hypertrophy by using a binary logistic stepwise regression method. Pearson correlation analysis was used to analyze the relationship between serum ATG9a and echocardiographic indices, and receiver operating characteristic (ROC) curves were plotted. We intended to analyze the diagnostic value of ATG9a for cardiac hypertrophy due to hypertension. We intended to analyze the diagnostic value of ATG9a for cardiac hypertrophy from hypertension. We present the following article in accordance with the STARD reporting checklist (available at https://apm.amegroups.com/article/ view/10.21037/apm-21-3936/rc).

\section{Methods}

\section{Research subjects}

In this study, 168 patients with hypertension in the Guizhou Medical University from February 2020 to September 2021 were selected and divided into an experimental group (cardiac hypertrophy group) and a normal group according to the results of ultrasound cardiogram examination. All procedures performed in this study involving human participants were in accordance with the Declaration of Helsinki (as revised in 2013). This study was approved by the Research Ethics Committee of Guizhou Medical University (No. 2021-247), and the patients or their families were informed of this study and signed the informed consent.

Inclusion criteria: (I) patients diagnosed with hypertension according to the diagnostic criteria of the Hypertension Prevention and Treatment Guidelines of China; (II) those diagnosed with cardiac hypertrophy but with normal myocardium according to the diagnostic criteria of Doppler ultrasound; (III) those not suffering from other serious organ or genetic diseases; (IV) patients without contraindications for examinations; and $(\mathrm{V})$ patients who had signed the informed consent forms.

Exclusion criteria: (I) patients with a history of liver and kidney dysfunction; (II) those with allergies to the experiment; (III) pregnant or breastfeeding patients; (IV) patients with secondary hypertension; (V) patients who were taking antihypertensive drugs; (VI) patients with autoimmune diseases; (VII) patients with serious diseases such as malignant tumors; (VIII) patients with congenital heart disease, heart failure, or other heart disease that may affect the results of this study; and (IX) patients with mental disorders.

\section{Ultrasonic imaging examination}

Ultrasonography was simultaneously performed in the 168 included patients. The examination instrument was the Philips Clear Vue350 Doppler ultrasound diagnostic instrument (Netherlands) with probe frequency of 3.5 MHz. During the examination, the patients were placed in a lateral or supine position to obtain the scanning images and determine the related indicators of cardiac hypertrophy: left ventricular ejection fraction (LVEF), interventricular septal thickness (IVST), left ventricular posterior wall thickness (LVPWT), left ventricular end-systolic dimension (LVESD), and left ventricular end-diastolic dimension (LVEDD). In addition, Left Ventricular Mass Index (LVMI) was calculated according to Eq. [1] as follows, where the body surface area is represented by $\mathrm{S}$.

$$
L V M=\frac{L V M}{S}
$$

Left Ventricular Mass (LVM) was calculated according to 
Eq. [2].

$$
L V M=0.8 \times 1.04 \times\left[\left(L V E D D+I V S T+L V P W T^{3}\right)-L V E D D^{3}\right]+0.6
$$

The body surface area $S$ was calculated according to Eq. [3].

$$
S=0.0061 \times H+0.0128 \times B M I-0.1529
$$

where $\mathrm{H}$ is the height of the patient, $\mathrm{BMI}$ is the body mass index, and the weight is represented by $W$. The BMI was calculated according to Eq. [4].

$$
B M I=\frac{W}{\mathrm{H}^{2}}
$$

\section{Acquisition of basic data}

The height and weight of the patients were measured, and the patients' basic information, such as age, hypertension course, and smoking history, was collected. Meanwhile, blood pressure was measured as below. Patients were forbidden to drink coffee or tea before the examination. During meditation (for about $5 \mathrm{~min}$ ), the patient's upper arm was kept at the same level as the heart. A desktop mercury sphygmomanometer was then used to determine the blood pressure after calibration. This step was performed twice with an interval of $2 \mathrm{~min}$. The diastolic and systolic blood pressure were recorded and then averaged.

\section{Detection of serum ATG9a}

Fasting venous blood was collected from the normal and experimental groups, and serum was obtained after centrifugation. The content of ATG9a in serum was detected according to enzyme linked immunosorbent assay (ELISA). After centrifugation, $100 \mu \mathrm{L}$ of serum was added into the detection plate well, and the standard substance and blank control group were set at the same time. The sample well was washed three times, and added with $50 \mu \mathrm{L}$ substrate $\mathrm{A}$ and $\mathrm{B}$ successively, followed by incubation at room temperature for approximately $15 \mathrm{~min}$. Finally, $50 \mu \mathrm{L}$ of stop solution was added to stop the reaction. The reaction plate was placed into a microplate reader (BioTek Instruments, USA, CYT3M) for determination, and relevant data were recorded to draw a standard curve. The serum ATG9a level was calculated according to the values obtained and the standard curve.

\section{Evaluation criteria}

In this study, three common indicators were selected to evaluate the efficacy of cardiac hypertrophy diagnosis from hypertension by ultrasound cardiogram in terms of accuracy, specificity, and sensitivity, as calculated using Eqs. [5], [6], and [7], respectively.

$$
\begin{aligned}
& \text { Accuracy }=\frac{A+B}{A+C+B+D}[5] \\
& \text { Specifity }=\frac{B}{C+B}[6] \\
& \text { Sensitivity }=\frac{A}{D+A}
\end{aligned}
$$

where, $A$ is true positive, indicating that the diagnosis result and the actual result are both true; $B$ is true negative, which means that the diagnostic and actual results are both negative; $\mathrm{C}$ is false positive, meaning that the diagnostic result is positive, but the actual result is negative; $\mathrm{D}$ is false negative, which means the actual result is positive and the diagnostic result is negative.

An ROC curve was used to represent the diagnostic efficiency of ATG9a, and the area under the ROC curve (AUC) was determined according to the ROC, and comparative analysis was conducted.

\section{Statistical analysis}

SPSS software (IBM Corporation, USA) was used for statistical analysis. Normally-distributed data were expressed as mean \pm standard deviation (mean $\pm \mathrm{SD}$ ). The $t$-test was used to represent measurement data, the Chisquare $\left(\chi^{2}\right)$ test was used to represent count data, and $\mathrm{P}<0.05$ indicated a statistical difference. The influencing factors of cardiac hypertrophy in hypertensive patients were analyzed by binary logistic regression, $\mathrm{P}<0.05$ indicated that the difference was statistically significant. Pearson correlation analysis was used to analyze the relationship between serum ATG9a and cardiac hypertrophy-related indicators.

\section{Results}

\section{Ultrasound imaging results of patients with cardiac bypertrophy from bypertension}

Patients with cardiac hypertrophy from hypertension were 

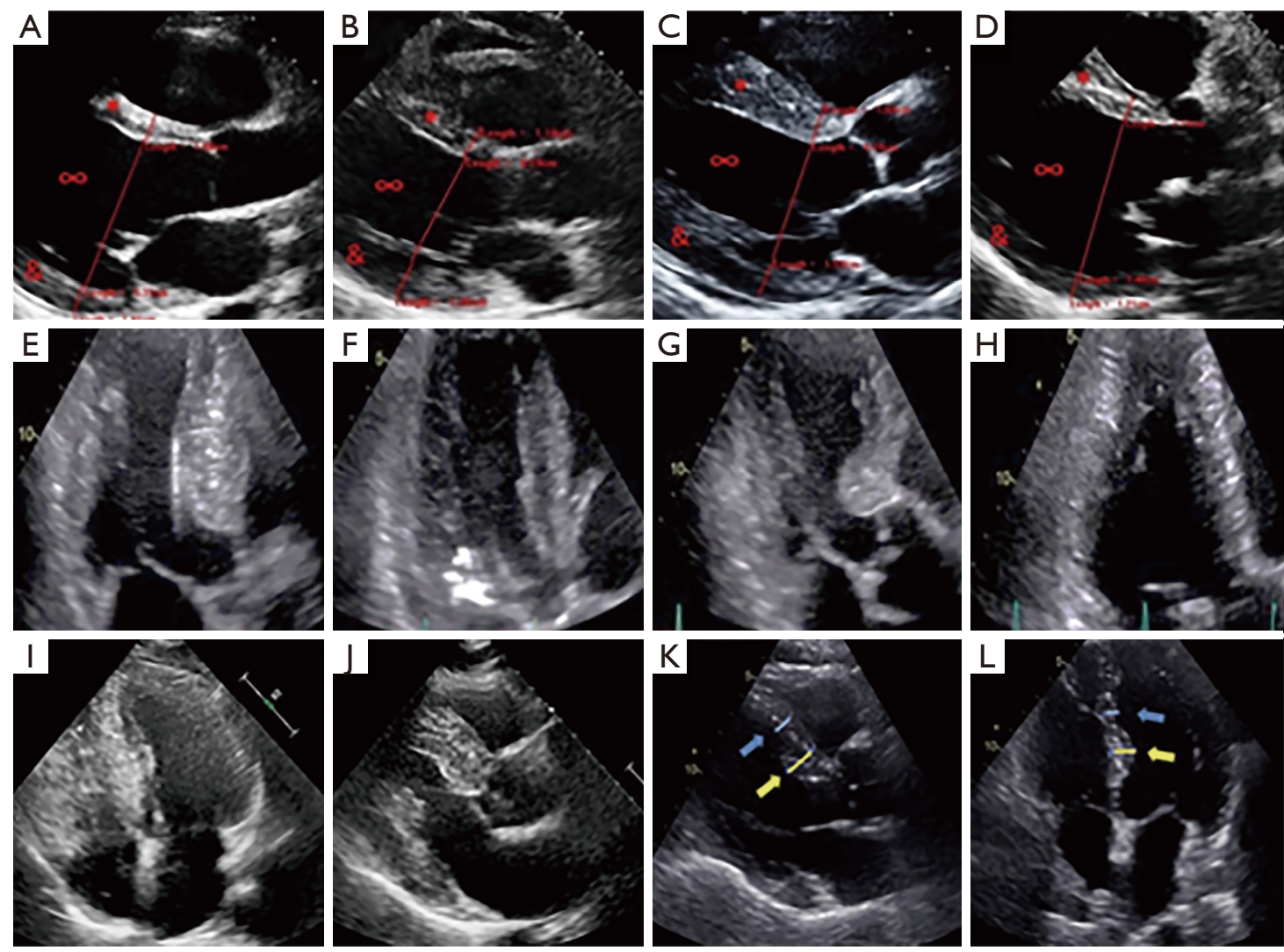

Figure 1 Ultrasound images of cardiac hypertrophy from hypertension (A-L). *, $\infty$, and * represented the position of the ventricular septum, anterior mitral valve, and inferior wall of the patient during the long axis section of the left ventricle measured by echocardiography, respectively. The areas with blue and yellow arrows all point to the patient's ventricular hypertrophy area.

examined by ultrasound imaging. Figure 1 (Figure $1 \mathrm{~A}$ as the control group) shows the ultrasound images of some of the patients. It was noted that many patients showed changes in the left ventricle, including narrowing of left ventricular cavity, thickening of left ventricular septum, thickening of left ventricular posterior wall, and symmetry of left ventricular hypertrophy.

\section{Comparison of the basic data of patients}

There were no significant differences in age, diastolic blood pressure, hypertension course, BMI, smoking history, and alcohol consumption history between the experimental and control groups $(\mathrm{P}>0.05)$. Systolic blood pressure in the experimental group was significantly lower than that in the normal group $(\mathrm{P}<0.05)$, as shown in Figure 2.

\section{Ultrasonic diagnosis-related indicators and ATG9a test results}

Doppler ultrasound was performed to measure the ultrasonic diagnosis-related indexes of the normal and experimental groups, and the expression level of ATG9a in serum was determined by ELISA. The results showed that, compared with the normal group, the level of ATG9a in the serum of the experimental group increased significantly $(\mathrm{P}<0.05)$. Additionally, the cardiac hypertrophy-related indexes of LVPWT, IVST, and LVMI also increased significantly, but LVEF significantly decreased $(\mathrm{P}<0.05)$. Meanwhile, there were no notable difference in the other 


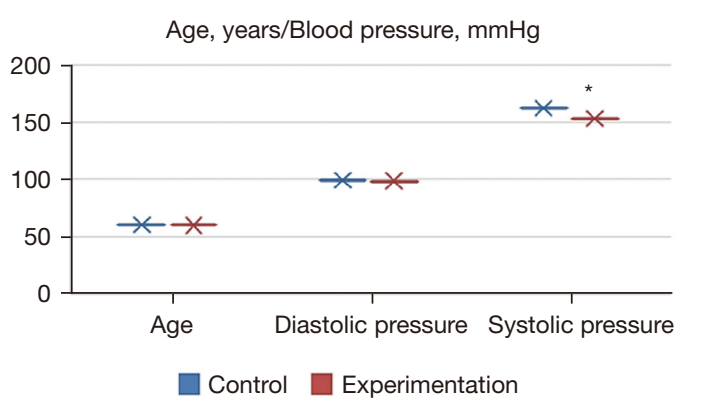

BMl, $\mathrm{kg} / \mathrm{m}^{2}$

/Hypertension/Smoking/drinking course, years

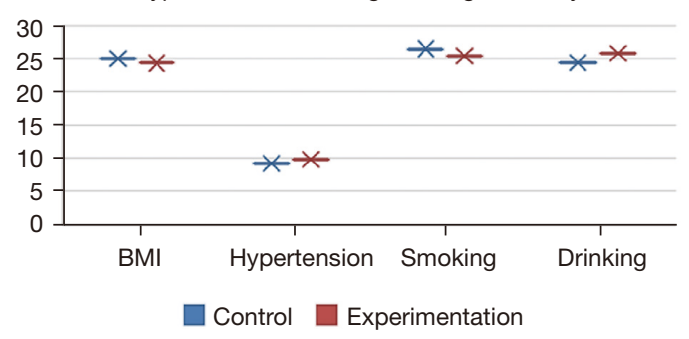

Figure 2 Comparison of the basic data between the two groups. * represented significant differences, $\mathrm{P}<0.05$. BMI, body mass index.

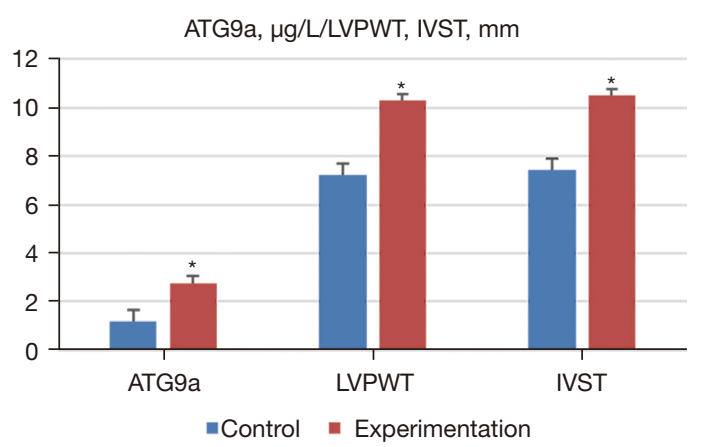

Figure 3 Comparison of serum ATG9a, LVPWT, and IVST between the two groups. * represented significant differences, $\mathrm{P}<0.05$. LVPWT, left ventricular posterior wall thickness; IVST, interventricular septal thickness.

cardiac hypertrophy-related indicators $(\mathrm{P}>0.05)$. The specific results are shown in Figures 3,4.

\section{Diagnostic results of serum ATG9a for cardiac bypertrophy from bypertension}

Using the results of ultrasound cardiogram as a reference, the accuracy, specificity, and sensitivity of ATG9a in the diagnosis of cardiac hypertrophy from hypertension were

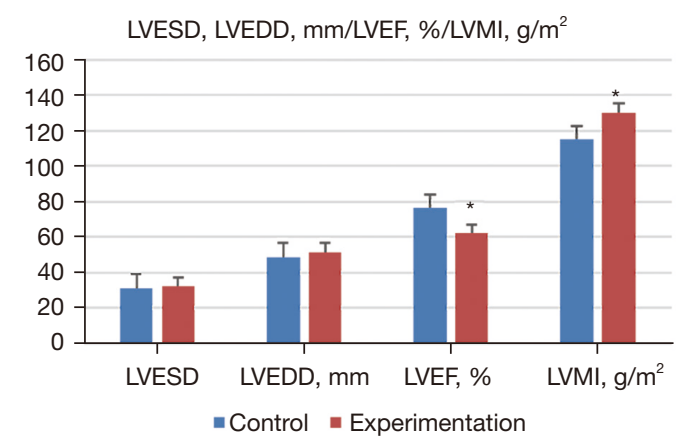

Figure 4 Comparison of LVESD, LVEDD, LVEF, and LVMI between the two groups. * represented significant differences, $\mathrm{P}<0$.05. LVESD, left ventricular end-systolic dimension; LVEDD, left ventricular end-diastolic dimension; LVEF, left ventricular ejection fraction; LVMI, left ventricular mass index.

$78.1 \%, 78.42 \%$, and $76.54 \%$, respectively, as shown in Figure 5 .

ROC curves were drawn according to the specificity and sensitivity of ATG9a as a marker for diagnosis, and the results are shown in Figure 6 below.

The AUC of ATG9a for the diagnosis of the cardiac hypertrophy from hypertension was 0.736 .

\section{Correlation analysis results of serum ATG9a and cardiac bypertrophy indicators}

The results of Pearson correlation analysis showed that the level of ATG9a in cardiac hypertrophy from hypertension was correlated with IVST, LVPWT, and LVEF $(\mathrm{P}<0.05)$, and that the other cardiac hypertrophy-related indicators were not correlated $(\mathrm{P}>0.05)$, as shown in Figure 7.

In addition, the level of ATG9a in the serum of cardiac hypertrophy from hypertension patients was positively correlated with IVST and LVPWT, and negatively correlated with LVEF, as shown in Figure 8.

\section{Discussion}

At present, chronic diseases have become important factors affecting the health and quality of life of patients, resulting in a considerable economic burden to the patients' families and society, as well as the public health and medical services system. Common chronic diseases include hypertension, coronary heart disease, and diabetes (40). Among these, the incidence of hypertension is the highest, and the heart has 


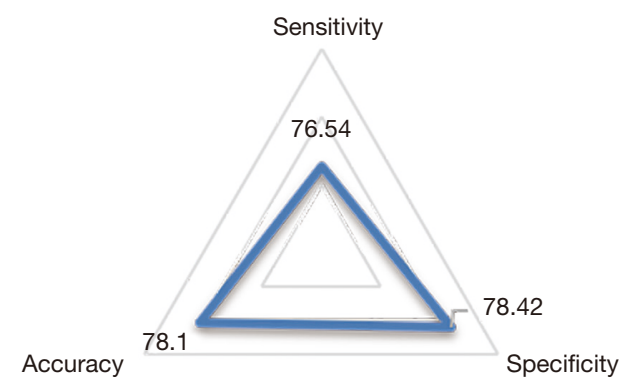

ATG9a, \%

Figure 5 Diagnostic value of serum ATG9a for cardiac hypertrophy from hypertension.

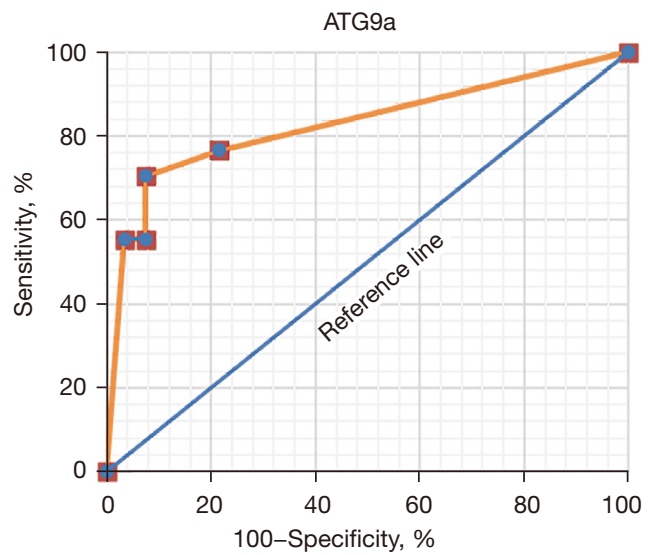

Figure 6 ROC curve of diagnosis of cardiac hypertrophy from hypertension by serum ATG9a. ROC, receiver operating characteristic.

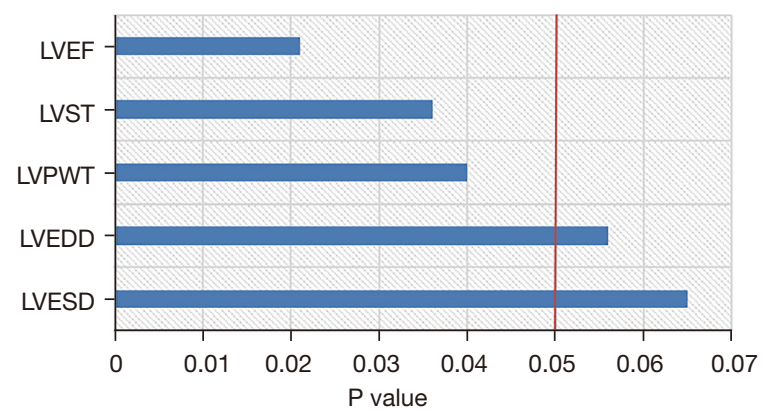

Figure 7 Correlation between serum ATG9a with indicators of cardiac hypertrophy. LVEF, left ventricular ejection fraction; IVST, interventricular septal thickness; LVPWT, left ventricular posterior wall thickness; LVEDD, left ventricular end-diastolic dimension; LVESD, left ventricular end-systolic dimension.

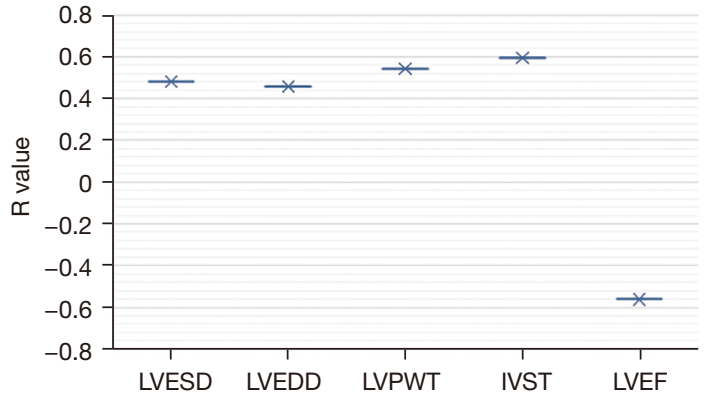

Figure 8 Positive and negative correlation between serum ATG9a and cardiac hypertrophy indicators. LVESD, left ventricular end-systolic dimension; LVEDD, left ventricular end-diastolic dimension; LVPWT, left ventricular posterior wall thickness; IVST, interventricular septal thickness; LVEF, left ventricular ejection fraction.

produced various adaptive compensatory responses to the long-term existence of high blood pressure. As an organ that promotes blood flow, the heart plays an important role in maintaining people's normal activities (41). With the improvement of living standards, the incidence of cardiovascular diseases is also increasing every year. In China, about 3 million people die of heart diseases every year, accounting for about $40 \%$ of the total causes of death. This has prompted discussion regarding early diagnosis and treatment among researchers (42). Among patients suffering from hypertension, about one-third of them also have left ventricular hypertrophy. Patients with hypertension are most likely to have cardiac dysfunction, resulting in hypertensive myocardial damage, most often manifested as hypertensive myocardial hypertrophy, which is one of the most common complications of hypertension, and most of them clinically manifest as left heart hypertrophy, the pathogenesis of which is still unclear. One study concluded that left heart hypertrophy is not only an independent risk factor for the development of hypertension, but also becomes an important risk factor for cardiovascular disease (43). It is held that ATG9a, as a multi-transmembrane protein, plays an important role in the formation of autolysosomes, and is abnormally expressed in patients with cardiac hypertrophy from hypertension. This may be because that it can inhibit cardiac hypertrophy. Hence, exploring its expression level in patients with cardiac hypertrophy from hypertension can provide a basis for the diagnosis of these patients. 
Ultrasound cardiogram is commonly used for the diagnosis of cardiac hypertrophy from hypertension. This study aimed to investigate the correlation between the occurrence of cardiac hypertrophy from hypertension with the expression of ATG9a. In this study, 168 patients with hypertension were selected and divided into an experimental group (cardiac hypertrophy group) and a normal group according to the results of ultrasound cardiogram examination. The serum ATG9a levels of patients in the two groups were detected. The association between ATG9a and cardiac hypertrophy from hypertension was analyzed by binary logistic stepwise regression. Pearson correlation analysis was used to analyze the relationship between serum ATG9a and ultrasound cardiogram indicators, and ROC curves were drawn to analyze the value of ATG9a in the diagnosis of cardiac hypertrophy from hypertension.

The results showed that there were no significant differences in age, diastolic blood pressure, hypertension course, BMI, smoking history, and drinking history between the experimental and normal groups $(\mathrm{P}>0.05)$. Binary logistic regression analysis showed that compared with the normal control group, ATG9a increased significantly $(\mathrm{P}<0.05)$, and systolic blood pressure decreased markedly in the experimental group. Also, the cardiac hypertrophyrelated indicators of LVPWT, IVST, and LVMI significantly increased, while LVEF significantly decreased $(\mathrm{P}<0.05)$, and other cardiac hypertrophy-related indicators exhibited no significant differences $(\mathrm{P}>0.05)$. These findings indicated that the expression level of ATG9a in the experimental group was increased, and the expression level of ATG9a in the serum may be related to the cardiac hypertrophy. The ROC curve analysis showed that the AUC of serum ATG9a was 0.736 , the sensitivity was $76.54 \%$, and the specificity was $78.42 \%$ in the diagnosis of cardiac hypertrophy from hypertension. The results of Pearson correlation analysis showed that ATG9a level of the cardiac hypertrophy from hypertension patients was positively correlated with LVPWT and IVST $(\mathrm{P}<0.05)$, and negatively correlated with LVEF $(\mathrm{P}<0.05)$.

It has been reported that ATG9a expression is increased in cardiac hypertrophy patients, which can inhibit cardiac hypertrophy by regulating cardiac autophagy. In addition, ATG9a and melatonin are positively correlated with the cardiac hypertrophy-related indicators, IVSD and LVPWD, which is consistent with the results of the present study (44). Therefore, serum ATG9a may be involved in the formation of cardiac hypertrophy in hypertensive patients, and patients with cardiac hypertrophy from hypertension had increased serum ATG9a levels. This is consistent with the clinical ultrasonic cardiogram diagnostic result, and ATG9a is expected to be a marker for early ultrasonic cardiogram diagnosis.

\section{Conclusions}

It was found that the expression level of ATG9a in the serum of the experimental group increased. In addition, ATG9a content was positively correlated with LVPWT and IVST, and negatively correlated with the LVEF of cardiac hypertrophy patients. The result that the serum ATG9a level increased in cardiac hypertrophy patients was consistent with the clinical ultrasonic cardiogram diagnosis result. However, some limitations in this study should be noted. The sample size is small, which will reduce the power of the study. In future research, an expanded sample size is necessary to strengthen the findings of the study. In conclusion, this study provides a basis for the diagnosis of cardiac hypertrophy from hypertension, and ATG9a is expected to be used as a marker in early cardiogram diagnosis.

\section{Acknowledgments}

Funding: This work was supported by National Natural Science Foundation of China (No. 81960315), Guiyang Science and Technology Bureau, Construction Contract [No. (2019) 9-1-29], and Youth Science and Technology Talent Development Project of School of Higher Education of Education of Guizhou Province [No. KY (2021) 186].

\section{Footnote}

Reporting Checklist: The authors have completed the STARD reporting checklist. Available at https://apm.amegroups. com/article/view/10.21037/apm-21-3936/rc

Data Sharing Statement: Available at https://apm.amegroups. com/article/view/10.21037/apm-21-3936/dss

Conflicts of Interest: All authors have completed the ICMJE uniform disclosure form (available at https://apm. amegroups.com/article/view/10.21037/apm-21-3936/coif). The authors have no conflicts of interest to declare. 
Ethical Statement: The authors are accountable for all aspects of the work in ensuring that questions related to the accuracy or integrity of any part of the work are appropriately investigated and resolved. All procedures performed in this study involving human participants were in accordance with the Declaration of Helsinki (as revised in 2013). This study was approved by the Research Ethics Committee of Guizhou Medical University (No. 2021-247), and the patients or their families were informed of this study and signed the informed consent.

Open Access Statement: This is an Open Access article distributed in accordance with the Creative Commons Attribution-NonCommercial-NoDerivs 4.0 International License (CC BY-NC-ND 4.0), which permits the noncommercial replication and distribution of the article with the strict proviso that no changes or edits are made and the original work is properly cited (including links to both the formal publication through the relevant DOI and the license). See: https://creativecommons.org/licenses/by-nc-nd/4.0/.

\section{References}

1. Slivnick J, Lampert BC. Hypertension and Heart Failure. Heart Fail Clin 2019;15:531-41.

2. Di Palo KE, Barone NJ. Hypertension and Heart Failure: Prevention, Targets, and Treatment. Heart Fail Clin 2020;16:99-106.

3. Lamirault G, Artifoni M, Daniel M, et al. Resistant Hypertension: Novel Insights. Curr Hypertens Rev 2020;16:61-72.

4. Lee JH, Kim KI, Cho MC. Current status and therapeutic considerations of hypertension in the elderly. Korean J Intern Med 2019;34:687-95.

5. Rossi GP, Bisogni V, Rossitto G, et al. Practice Recommendations for Diagnosis and Treatment of the Most Common Forms of Secondary Hypertension. High Blood Press Cardiovasc Prev 2020;27:547-60.

6. Tziomalos K. Secondary Hypertension: Novel Insights. Curr Hypertens Rev 2020;16:11.

7. Manosroi W, Williams GH. Genetics of Human Primary Hypertension: Focus on Hormonal Mechanisms. Endocr Rev 2019;40:825-56.

8. van den Born BH, Lip GYH, Brguljan-Hitij J, et al. ESC Council on hypertension position document on the management of hypertensive emergencies. Eur Heart J Cardiovasc Pharmacother 2019;5:37-46.
9. Hisamatsu T, Segawa H, Kadota A, et al. Epidemiology of hypertension in Japan: beyond the new 2019 Japanese guidelines. Hypertens Res 2020;43:1344-51.

10. Ondimu DO, Kikuvi GM, Otieno WN. Risk factors for hypertension among young adults (18-35) years attending in Tenwek Mission Hospital, Bomet County, Kenya in 2018. Pan Afr Med J 2019;33:210.

11. Gupta A, Prince M, Bob-Manuel T, et al. Renal denervation: Alternative treatment options for hypertension? Prog Cardiovasc Dis 2020;63:51-7.

12. Macumber I, Flynn JT. Does treatment-resistant hypertension exist in children? A review of the evidence. Pediatr Nephrol 2020;35:969-76.

13. Loutradis C, Sarafidis P. Pharmacotherapy of hypertension in patients with pre-dialysis chronic kidney disease. Expert Opin Pharmacother 2020;21:1201-17.

14. Khalid K, Padda J, Ismail D, et al. Correlation of Coronary Artery Disease and Left Ventricular Hypertrophy. Cureus 2021;13:e17550.

15. Yang Y, Yu WW, Yan W, et al. Decorin Induces Cardiac Hypertrophy by Regulating the CaMKII/MEF-2 Signaling Pathway In Vivo. Curr Med Sci 2021;41:857-62.

16. Caddigan S, Granlund B. Anesthesia For Patients With Pulmonary Hypertension Or Right Heart Failure. 2021 Oct 11. In: StatPearls (Internet). Treasure Island (FL): StatPearls Publishing; 2021 Jan. PMID: 34283437.

17. Mondaca-Ruff D, Araos P, Yañez CE, et al. Hydrochlorothiazide Reduces Cardiac Hypertrophy, Fibrosis and Rho-Kinase Activation in DOCA-Salt Induced Hypertension. J Cardiovasc Pharmacol Ther 2021;26:724-35.

18. Tang M, Chen Y, Sun F, et al. The Dose-Dependent Effects of Spironolactone on TGF- $\beta 1$ Expression and the Vulnerability to Atrial Fibrillation in Spontaneously Hypertensive Rats. Cardiol Res Pract 2021;2021:9924381.

19. Liang Y, Xu Y, Ding L, et al. Urotensin II Induces Cardiac Fibrosis through the TGF- $\beta /$ Smad Signaling Pathway during the Development of Cardiac Hypertrophy. Int Heart J 2021;62:1135-44.

20. Crea F. The ESC Guidelines on heart failure, sacubitrilvalsartan in resistant hypertension, and new therapeutic targets in myocardial hypertrophy. Eur Heart J 2021;42:3581-5.

21. Oknińska M, Zambrowska Z, Zajda K, et al. Right ventricular myocardial oxygen tension is reduced in monocrotaline-induced pulmonary hypertension in the rat 
and restored by myo-inositol trispyrophosphate. Sci Rep 2021;11:18002.

22. Lin CY, Shibu MA, Wen R, et al. Leu27 IGF-II-induced hypertrophy in $\mathrm{H} 9 \mathrm{c} 2$ cardiomyoblasts is ameliorated by saffron by regulation of calcineurin/NFAT and CaMKII $\delta$ signaling. Environ Toxicol 2021;36:2475-83.

23. Yuchi Y, Suzuki R, Kanno H, et al. Right Ventricular Myocardial Adaptation Assessed by Two-Dimensional Speckle Tracking Echocardiography in Canine Models of Chronic Pulmonary Hypertension. Front Vet Sci 2021;8:727155.

24. Frenzel S, Wittfeld K, Bülow R, et al. Cardiac Hypertrophy Is Associated With Advanced Brain Aging in the General Population. J Am Heart Assoc 2021;10:e020994.

25. Potnuri AG, Purushothaman S, Saheera S, et al. Mitotargeted antioxidant prevents cardiovascular remodelling in spontaneously hypertensive rat by modulation of energy metabolism. Clin Exp Pharmacol Physiol 2022;49:35-45.

26. Kwan ED, Vélez-Rendón D, Zhang X, et al. Distinct time courses and mechanics of right ventricular hypertrophy and diastolic stiffening in a male rat model of pulmonary arterial hypertension. Am J Physiol Heart Circ Physiol 2021;321:H702-15.

27. Sunagawa Y, Funamoto M, Shimizu K, et al. Curcumin, an Inhibitor of p300-HAT Activity, Suppresses the Development of Hypertension-Induced Left Ventricular Hypertrophy with Preserved Ejection Fraction in Dahl Rats. Nutrients 2021;13:2608.

28. Ho MY, Wang CY. Role of Irisin in Myocardial Infarction, Heart Failure, and Cardiac Hypertrophy. Cells 2021;10:2103.

29. Olivares-Silva F, De Gregorio N, Espitia-Corredor J, et al. Resolvin-D1 attenuation of angiotensin II-induced cardiac inflammation in mice is associated with prevention of cardiac remodeling and hypertension. Biochim Biophys Acta Mol Basis Dis 2021;1867:166241.

30. Bello H, Norton GR, Peterson VR, et al. Hemodynamic and Functional Correlates of Concentric vs. Eccentric LVH in a Community-Based Sample With Prevalent Volume-Dependent Hypertension. Am J Hypertens 2021;34:1300-10.

31. Labbé V, Ederhy S, Lapidus N, et al. Transesophageal echocardiography for cardiovascular risk estimation in patients with sepsis and new-onset atrial fibrillation: a multicenter prospective pilot study. Ann Intensive Care
2021;11:146.

32. Baweja P, Sweeney MJ, López-Candales A. A Reminder That Stress Echocardiography Is Useful in Diagnosing Myocardial Ischemia in Nonobstructive Coronary Artery Disease: Case Series. Cureus 2021;13:e17763.

33. Han KN, Ma XT, Yang SW, et al. Intracardiac echocardiography in the diagnosis and closure of patent foramen ovale. J Geriatr Cardiol 2021;18:697-701.

34. Espinola-Zavaleta N, Fernández-Badillo V, SolorzanoPinot E. New approach of contrast echocardiography: differential diagnosis between pulmonary thrombi and tumors in the pulmonary arteries. Int J Cardiovasc Imaging 2021. [Epub ahead of print].

35. Xu X, Su YL, Shi JY, et al. MicroRNA-17-5p Promotes Cardiac Hypertrophy by Targeting Mfn2 to Inhibit Autophagy. Cardiovasc Toxicol 2021;21:759-71.

36. Ott C, Jung T, Brix S, et al. Hypertrophy-Reduced Autophagy Causes Cardiac Dysfunction by Directly Impacting Cardiomyocyte Contractility. Cells 2021;10:805.

37. Guardia CM, Jain A, Mattera R, et al. RUSC2 and WDR47 oppositely regulate kinesin-1-dependent distribution of ATG9A to the cell periphery. Mol Biol Cell 2021;32:ar25.

38. daSilva-deAbreu A, Alhafez BA, Lavie CJ, et al. Interactions of hypertension, obesity, left ventricular hypertrophy, and heart failure. Curr Opin Cardiol 2021;36:453-60.

39. Ba L, Gao J, Chen Y, et al. Allicin attenuates pathological cardiac hypertrophy by inhibiting autophagy via activation of PI3K/Akt/mTOR and MAPK/ERK/mTOR signaling pathways. Phytomedicine 2019;58:152765.

40. Fan W, Zhang B, Wu C, et al. Plantago asiatica L. seeds extract protects against cardiomyocyte injury in isoproterenol- induced cardiac hypertrophy by inhibiting excessive autophagy and apoptosis in mice. Phytomedicine 2021;91:153681.

41. Giorgione V, O'Driscoll J, Coutinho CM, et al. Peripartum echocardiographic changes in women with hypertensive disorders of pregnancy. Ultrasound Obstet Gynecol 2021. [Epub ahead of print].

42. Meijles DN, Cull JJ, Cooper STE, et al. The anticancer drug dabrafenib is not cardiotoxic and inhibits cardiac remodelling and fibrosis in a murine model of hypertension. Clin Sci (Lond) 2021;135:1631-47.

43. Yildiz M, Oktay AA, Stewart MH, et al. Left ventricular hypertrophy and hypertension. Prog Cardiovasc Dis 
2020;63:10-21.

44. Gao M, Hu F, Hu M, et al. Sophoricoside ameliorates cardiac hypertrophy by activating AMPK/mTORC1- mediated autophagy. Biosci Rep 2020;40:BSR20200661.

(English Language Editor: A. Kassem)

Cite this article as: Li S, Zhao L, Zhang B, Yuan Y, Cao $\mathrm{H}, \mathrm{Yu} \mathrm{Z}$. Ultrasound cardiogram-based diagnosis of cardiac hypertrophy from hypertension and analysis of its relationship with expression of autophagy-related protein. Ann Palliat Med 2022;11(2):684-694. doi: 10.21037/apm-21-3936 